\title{
THE PROBLEMATIC ALLEVIATING SUFFERING ARGU- MENT WHY GENETIC ENGINEERING IS UNNECESSARY
}

\section{Lorena Dürnholz}

\begin{abstract}
Few topics are as controversial today as genetic engineering, which would allow parents to screen and subsequently modify the genome of their unborn children. Advocates commonly argue that genetic engineering is desirable because it could prevent diseases and disabilities and this way alleviate suffering. However, this argument relies on a simplistic and one-sided view of health. By adopting a more relational perspective, it becomes clear that genetic engineering is unnecessary, firstly because the amount of suffering associated with the genetic conditions it could prevent is much lower than one might expect, and secondly because the suffering that might nevertheless be present can also be alleviated by other means, which are to be preferred.
\end{abstract}

\section{Introduction}

Few topics are as controversial today within the field of bioethics as genetic engineering. This is a procedure that allows parents to genetically modify their children before they are even born. In fact, in light of the considerable recent advances in the areas of molecular biology and reproductive medicine, such genetic engineering seems no longer mere science-fiction. For instance, in-vitro 
fertilization and preimplantation genetic diagnosis have become routinized medical procedures, and much research is currently being dedicated to the sequencing of the human genome as well as the development of gene therapy (e.g. Lander et al., 2001; Thomas, Ehrhardt, \& Kay, 2003). As a result, parents might, indeed, soon be able to use these techniques in order to screen and subsequently modify the genome of their future children (Ewing, 1987). This would allow parents to correct genetic mutations that are deemed undesirable, such as those associated with genetic diseases or disabilities, or even to select and potentially enhance traits that are considered advantageous. While proponents embrace this future perspective with much hope and enthusiasm, genetic engineering clearly also raises a variety of ethical concerns, which need to be examined carefully in order to ensure that this technology, should it ever be developed, will be used in a responsible and desirable manner.

One of the most prominent arguments commonly brought forward by advocates of genetic engineering holds that genetic engineering is beneficial because it alleviates human suffering (Suckling, Root, \& Millares-Martin, 2000), which is defined as any state of serious distress that threatens the physical, emotional, mental, spiritual or social intactness of a person (Oreopoulos, 2005). In fact, by means of genetic engineering, parents could correct genetic mutations that would otherwise result in chronic diseases or disabilities. Proponents claim that, as such chronic diseases and disabilities are necessarily associated with a considerable amount of suffering, as well as a decreased quality of life, preventing such disabilities will alleviate human suffering. According to these advocates of genetic engineering, this constitutes a morally desirable possibility which should be embraced (Suckling, Root, \& Millares-Martin, 2000).

However, this argument is problematic, because the assumption that genetic diseases and disabilities necessarily come with an increased amount of suffering relies on a simplistic view of health, which cannot do justice to the complex experiences of those affected by such chronic diseases and disabilities. By adopting a more relational approach, defining health not simply as the absence of disease but as the outcome of a complex interplay between both medical and contextual factors, it becomes clear that genetic engineering is unnecessary for two reasons. Firstly, the amount of suffering associated with those genetic conditions that could be prevented by genetic engineering is much lower than one might expect, if present at all. Secondly, the suffering these individuals might nevertheless experience can also be alleviated by other means, which are to be preferred as they also benefit the majority of diseased or disabled individuals whose impairments result from non-genetic factors. 


\section{Why Genetic Engineering is Unnecessary}

At first glance, it might seem appealing to believe that genetic engineering is desirable because it could prevent a considerable amount of suffering. However, this argument only holds if those genetic conditions which could be prevented by genetic engineering are, indeed, necessarily associated with a high amount of suffering, and if this suffering cannot be alleviated by other means that are preferable over genetic engineering. Both of these assumptions are questionable, as they rely on the highly contested medical, or attributional, model of health and disease. According to this model, health is simply defined as the absence of disease and thus as a bodily property that is, or is not, possessed by a person (Amzat \& Razum, 2014; Kagawa-Singe, 1993). In fact, after the scientific revolution in the $17^{\text {th }}$ and $18^{\text {th }}$ century, scientists in the medical field became increasingly interested in observing, classifying, and causally explaining the various symptoms of diseases. This way, the focus of attention gradually shifted away from the broader social environment of the patient towards the internal workings of the body, and health became seen as an individual rather than a social matter (Bury, 2005). As a result, the medical model considers diseases or disabilities as a problem of the diseased or disabled person only, caused by physical or mental impairments. These impairments are thought to impose severe limitations and suffering on the concerned individual, who requires sustained support and medical care (Bury, 2005). Moreover, since diseases and disabilities are considered an individual matter, management of the condition is exclusively directed towards the diseased or disabled individual, and treatment aims at a cure or, if this is not possible, at the adjustment and behavioral change of the person concerned(Langtree, 2015). From this perspective, genetic engineering seems desirable, as it would liberate an individual from the burdens of the physical impairments that she would necessarily have experienced had she been born with a given disease or disability.

However, the attributional view of health as outlined above is strongly criticized today for not doing justice to the complex nature of health and disease. Many advocates of the disabilities rights movement, for instance, point out that it is wrong to assume that physical or mental impairments, such as those resulting from genetic conditions, are necessarily associated with a decreased quality of life and an increased amount of suffering (World Health Organization, 2011). In fact, a growing body of empirical evidence shows that many individuals with chronic diseases or disabilities do not consider themselves unhealthy and do not report a high degree of suffering. For instance, $40 \%$ of people with severe disabilities who responded to the 2007-2008 Australian National Health Survey rated their health as good, very good, or even excellent (World Health Organization, 
2011). Moreover, studies found that the average quality of life of people with cystic fibrosis (CF) does not differ from that of healthy controls in spite of the various challenges encountered by CF patients, including multiple medications, chest drainage maneuvers, frequent hospitalizations, and an average life expectancy of only 37.4 years (Boslet, 2011). Similarly, when interviewing cancer patients about their perceived health, Kagawa-Singe (1993) found that 49 out of 50 individuals nevertheless perceived themselves as being healthy, and that they were able to maintain a sense of self-integrity as individuals despite their physical challenges (Kagawa-Singe, 1993). By far, the clearest evidence that disabilities do not necessarily entail unbearable suffering comes from a study assessing the self-perception of 284 people with Down syndrome all across the United States. In this study, $99 \%$ of respondents indicated that they were very happy with their lives, 97\% liked who they are, and 96\% liked how they look (Skotko, Levine, \& Goldstein, 2011).

Of course, these studies did not cover all genetic conditions, and some individuals with chronic diseases or disabilities might nevertheless consider themselves as suffering to a certain extent. Nevertheless, these findings indicate that the relation between physiological and perceived health is much more complex than suggested by the medical or attributional model of health and disease on which advocates of genetic engineering base their argument. More specifically, these studies show that the extent to which diseased or disabled people are able to cope with their health challenges is not only determined by their body, for if this was the case, all individuals with a given disability would be facing an equally high amount of suffering. Rather, these studies indicate that the extent to which individuals suffer from a chronic disease or disability also depends on other factors. This calls for a more social and relational approach to disability. In fact, advocates of such a social conception of the term, including many disabled people themselves, argue that disability should be understood as a dynamic interplay between health conditions and contextual factors (World Health Organization, 2011). In this model, the limitations experienced by disabled people are thought to be linked not only to their medical condition, but also to the barriers created by their environment and the attitudes of the people they encounter. Thus, disability should no longer be considered a mere attribute of the disabled person, and diseased or disabled people are no longer viewed as being disabled by their body only, but also by society (World Health Organization, 2011). Management of the disability, then, is not exclusively directed towards the disabled individual, but also towards society as a whole, so that the societal barriers encountered by this individual can be reduced. This could be achieved, for instance, by developing more effective therapeutic means to better manage the 
physical pain that might come with certain genetic conditions, and by changing the societal attitudes towards people with chronic diseases or disabilities. Eventually, this could create a more tolerant environment, in which people with genetic conditions would no longer experience their physical impairments as severe limitations (World Health Organization, 2011). Moreover, building a more tolerant society can not only benefit those individuals affected by genetic conditions, who constitute a minority, but it will also facilitate? the lives of the majority of diseased and disabled people who acquire their disability in the course of their lives and whose condition could never have been prevented by means of genetic engineering.

Together, the empirical evidence along with a more relational approach to disease and disability show that genetic engineering is unnecessary for two reasons. Firstly, the suffering associated with those genetic conditions it could prevent is very small to begin with, if present at all. As the quality of life of many diseased or disabled individuals is as high as that of healthy people, genetic engineering is already unnecessary in many cases. Secondly, even if some individuals with chronic genetic conditions might still be suffering to a certain extent today, it becomes clear by adopting a more relational perspective on health that their suffering could also be alleviated in the future by alternative means which do not require genetic engineering. Rather than using genetic engineering in order to prevent the existence of genetically diseased or disabled individuals on the wrong assumption that they will necessarily be suffering, the quality of life of existent individuals with diseases or disabilities could be further increased, if necessary, by ensuring that their special needs are met and that they face as few limitations as possible. This relational view on health makes genetic engineering not only appear unnecessary, but an alternative approach which aims at creating a more tolerant society is even preferable, as it will benefit not only genetically diseased and disabled individuals but also the many people affected by non-genetic impairments.

\section{The Problem of Severe Early Onset Diseases}

Even though some advocates of genetic engineering acknowledge that the suffering associated with many genetic conditions might be lower than expected or even absent in many cases, they often point out that there are nevertheless certain diseases which necessarily impose an unbearable amount of suffering on the affected individual and those surrounding them. For instance, Tay-Sachs is a severe early onset disease that causes both physical and mental retardation and 
eventually results in death between age four and five (Boslet, 2011). Proponents of genetic engineering claim that in such cases, the impairment of the child's quality of life and the suffering inflicted on both the child and its parents are so great that non-existence is to be preferred over existence, and that genetic interventions should consequently be used in exceptional cases to prevent such children from being born (Boslet, 2011). While the birth of a child with Tay-Sachs or another severe early onset disease indisputably constitutes a highly challenging situation, four points have to be emphasized here. The first two focus on the experience of the child, the third addresses the potential suffering of the parents, and the fourth concerns the practical and moral problems concerning the suggestion to allow genetic engineering only in rare exceptions.

Firstly, advocates who plead in favor of genetic engineering to prevent severe early onset diseases assume that a short life is necessarily associated with a higher degree of suffering than a long life, but this assumption has to be questioned. Of course, every parent wants its child to be able to grow into an adult with a fulfilled and ideally a very long life. However, there are no grounds to objectively assert that a short life is necessarily less valuable for the affected child than a longer life would have been. For instance, toddlers are unable to understand the concept of death. In fact, most children realize that they themselves will eventually die only when they reach school age (University of Rochester Medical Center, 2016). Thus, there is no reason to believe that children affected by a severe early onset disease, who unfortunately have to die before they even understand what death means, are suffering from the knowledge that they will die sooner and have a shorter life than others - or as a mother once said in an interview about her son affected by Tay-Sachs: "He never worried, because he didn't know how" (Braiker, n.d., para. 16). Secondly, given the recent advances with regards to palliative care and the management of pain, the physical suffering that might be experienced by affected children can already be alleviated tremendously today. Additionally, psychological support as well as a loving and caring environment might equally contribute to making the child's short life as enjoyable as possible. In sum, the claim brought forward by advocates of genetic engineering that non-existence is to be preferred over existence in the case of diseases such as Tay-Sachs is highly questionable.

When it comes to the suffering of the parents, it cannot be denied that parents of children with severe early onset diseases have to go through a very difficult process of having to care for and then let go of their diseased child. However, just as for the child, psychological support is already available today and can successfully help these parents in dealing with this situation. Moreover, despite the challenges they encounter, many parents of children with severe early onset dis- 
eases such as Tay-Sachs are nevertheless grateful for the short yet valuable time spent with their children, and they give meaning to the disease by incorporating it into their identity. For instance, in an interview, a mother of a son who died of Tay-Sachs explained: "Sometimes he would do something that would make me laugh. He was gorgeous and sweet. You could snuggle with him and take him places. All of that was happy. [...] I have a great life. It's a sad, complicated, beautiful and strange life. It's mine. [...] I'm glad I was his mother. I just wish I had been able to be it for longer" (Braiker, n.d., para. 30). Finally, parents who are at risk to have a child with a severe early onset disease and who know that they would not be able to cope with this situation can already make use of alternatives that are available today and do not require genetic engineering. For instance, parents can, in most cases, get a healthy child by means of gamete donation, adoption or a combination of in-vitro fertilization and preimplantation genetic diagnosis, though the latter admittedly also comes with ethical concerns that need to be examined. Together, the available psychological support for parents, the ability of many parents to cope surprisingly well with such a difficult situation, and the ample available alternatives once again make genetic engineering unnecessary.

Lastly, the suggestion of many advocates to allow genetic engineering only in a small number of exceptional cases, such as severe early onset diseases, is problematic for several reasons. In fact, it would require us to decide which diseases count as sufficiently 'bad' to be entitled to such an exceptional status. In order to do so, one would have to decide upon the criteria in terms of which diseases would be classified as causing more or less suffering and as being more or less worthy to be prevented. However, such a classification is both practically unfeasible and morally unacceptable. It is unfeasible because it would require us to objectively assess, weigh, and compare the level of suffering that is associated with these diseases. It is simply impossible to accomplish this, as different diseases come with radically different challenges, and as every individual affected by a given disease experiences this disease and the suffering that might result from it in his or her own way. Consequently, any distinction between diseases that should and those that should not be prevented by genetic engineering would be arbitrary. Additionally, such exceptions are not only unfeasible but also morally questionable, as such a distinction would convey the message that some diseases are acceptable while others are seen as 'condemning' children to a life that is deemed not worth living and that should consequently be prevented. Thus, not only is the exceptional use of genetic engineering to prevent severe early onset diseases unnecessary in many cases, as shown above, but it is also unfeasible and even morally questionable. 


\section{Fixing society rather than our genome}

Even though it might be tempting to believe that genetic engineering is desirable because it could alleviate suffering, this argument is problematic because it approaches the problem of suffering from a simplistic and one-sided perspective. Indeed, this view ignores the subjective and multifaceted nature of suffering and the complex experience of those individuals affected by conditions that genetic engineering could prevent. Empirical evidence suggests that many chronically diseased or disabled people do, in fact, not suffer more than healthy people. As a result, genetic engineering is already unnecessary in a majority of cases today. This even applies to severe early onset diseases, because a shorter life is not less valuable than a longer life, the suffering of both the affected children and their parents can already be alleviated by other means, and there are alternatives available for parents who know that they could not cope with a child diagnosed with such a disease.

Moreover, by adopting a more relational approach to health, it becomes clear that being healthy means much more than just having the 'right' genome or not having a given disease. Of course, the medical challenges resulting from genetic conditions can, and should, not be ignored. However, the various social and contextual factors that might influence the suffering experienced by diseased and disabled individuals have to be acknowledged as well. Rather than trying to fix an individual's genome on the wrong assumption that genetic mutations necessarily cause unbearable suffering, the suffering of genetically diseased or disabled individuals can also be alleviated if society adapts to the special needs of these individuals, for instance by investing into a well-functioning health care system and by creating a more tolerant social environment. Not only would genetic engineering be completely unnecessary in such a society, but trying to alleviate suffering by adjusting our attitudes rather than our genes is even preferable, as everyone will ultimately benefit from a more open and tolerant society in which diseased or disabled people are included and their existence is not prevented in the first place. Since only the minority of diseases and disabilities is genetically determined, society will always have to deal with diseased and disabled individuals, and the less disabilities are regarded as abnormalities that could, or even should, be corrected, the less suffering those who are disabled or diseased as a result of both genetic and non-genetic factors will experience.

Overall, genetic engineering approaches suffering from the wrong angle, is in many cases already unnecessary, and can be made fully unnecessary in the future if society adopts a more relational approach to health and disease. Consequently, it seems much more desirable to use the effort, time, and money 
which is currently being dedicated to the development of genetic engineering instead for much more pressing and necessary goals, such as the establishment of precisely such a society in which the development of genetic engineering would eventually no longer be needed at all.

\section{References}

Amzat, J., \& Razum, O. (2014). Medical sociology in Africa. Cham: Springer International Publishing. Bosslet, G. T. (2011). Parental procreative obligation and the categorisation of disease: The case of cystic fibrosis. Journal of Medical Ethics, 37(5), 280-284.

Braiker, B. (n.d.). Q\&A: Emily Rapp on parenting without a future. Retrieved April 15, 2016, from http://www.parenting.com/article/emily-rapp-tay-sachs

Bury, M. (2005). Health and illness. Cambridge: Polity.

Ewing, C. (1987). Designer genes: Genetic engineering and eugenics. Retrieved April 15, 2016, from http://www.finrrage.org/wp-content/uploads/2016/03/Designer_Genes_Ewing1987.pdf

Kagawa-Singe, M. (1993). Redefining health: Living with cancer. Social Science \& Medicine, 37(3), 295-304.

Lander, E. S., Linton, L. M., Birren, B., Nusbaum, C., Zody, M. C., Baldwin, J., ... \& Funke, R. (2001). Initial sequencing and analysis of the human genome. Nature, 409(6822), 860-921.

Langtree, I. (2015). Definitions of the models of disability. Retrieved April 15, 2016, from http:// www.disabled-world.com/definitions/disability-models.php

Oreopoulos, D. G. (2005). Is there meaning in suffering?. Humane Medicine, 5(2). Retrieved April 15, 2016, from http://www.hektoeninternational.org/documents/ISTHEREMEANINGINSUFFERING.pdf

Skotko, B. G., Levine, S. P., \& Goldstein, R. (2011). Self-perceptions from people with Down Syndrome. American Journal of Medical Genetics, 155(10), 2360-2369.

Suckling, R., Root, S., \& Millares-Martin, P. (2000). Eugenics debate. British Medical Journal, 320(7238), 873.

Thomas, C. E., Ehrhardt, A., \& Kay, M. A. (2003). Progress and problems with the use of viral vectors for gene therapy. Nature Reviews Genetics, 4(5), 346-358.

University of Rochester Medical Center (2016). A child's concept of death. Retrieved April 15, 2016, from https://www.urmc.rochester.edu/Encyclopedia/Content.aspx?ContentTypeID=90\&ContentID=P0304 4

World Health Organization (2011). Chapter 1 Understanding disability, world report on disability. Retrieved April 15, 2016, from http://www.who.int/disabilities/world_report/2011/report/en/ 